\title{
Functional foods and bioactive molecules with relevance to health and chronic disease
}

\section{Ian James Martins ${ }^{1,2,3}$}

${ }^{1}$ Centre of Excellence in Alzheimer's Disease Research and Care' School of Medical and Health Sciences, Edith Cowan University, 270 Joondalup Drive, Joondalup, 6027, Australia; ${ }^{2}$ School of Psychiatry and Clinical Neurosciences, The University of Western Australia, Nedlands, 6009, Australia; ${ }^{3}$ McCusker Alzheimer's Research Foundation, Hollywood Medical Centre, 85 Monash Avenue, Suite 22, Nedlands, 6009, Australia

Corresponding author: Dr Ian Martins, School of Medical Sciences, Edith Cowan University, 270 Joondalup Drive, Joondalup, Western Australia 6027, Australia

Submission Date: September $1^{\text {st }}, 2017$, Acceptance Date: October $27^{\text {th }}$, 2017, Publication Date: October $31^{\text {st }}, 2017$

Citation: Martins IJ, Functional Foods and Active molecules with relevance to Health and Chronic disease: Editorial. Functional Foods in Health and Disease 2017; 7(10): 849-852. https://doi.org/10.31989/ffhd.v7i10.387

Keywords: calorie sensitive gene, nutrition, mitophagy, senescence, active biomolecules, diabetes, functional foods, caffeine, chronic disease

\section{EDITORIAL}

In recent years, food scientists have published several articles which state how functional foods contain known, effective biologically-active compounds [1]. Some of these biologically-active, non-toxic compounds have advantageous physiological effects [1] and could play an important role in current global chronic disease epidemics. Functional food science [2] has become essential to the treatment strategies for various diseases, such as obesity, diabetes, and neurodegenerative diseases (Parkinson's disease and Alzheimer's disease) [3]. These diseases have significantly increased in this century. For example, the incidence of diabetes has been predicted to increase to $21 \%$ and non-alcoholic fatty liver disease (NAFLD) to $40 \%$ of the global population by 2050.

Nutritional science provides functional foods with the required amount of natural antioxidants, vitamins, fats, proteins, carbohydrates, and other components required to maintain the immune system and for the survival of cells [4-6]. The majority of health benefits and physiological activities attributed to various functional foods and nutrients such as polyphenols which are important for brain health [7-9] require more scientific research to understand the role of caffeine as a dietary supplement [10,11]. Defective caffeine metabolism [12-14] with relevance to the global NAFLD epidemic is linked to the defective adipose tissue-liver cross talk [15] and brain interaction (Figure 1). Furthermore, caffeine may interfere with novel functional food therapy [14] with relevance to brain function and the adipose tissue crosstalk [16, 17]. 


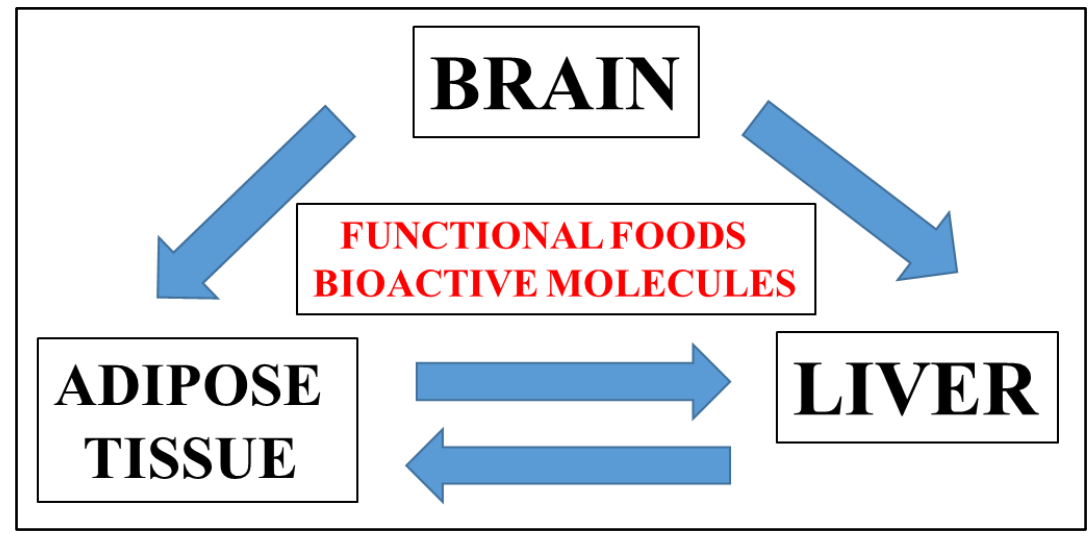

Figure 1. The use of functional foods and bioactive molecules to maintain connections between the brain and the adipose tissue-liver crosstalk has accelerated with relevance to the global NAFLD and diabetes epidemic. Nutritional science has become important to the brain and liver function with caffeine metabolism important to the function of bioactive molecules with relevance to the global NAFLD and diabetes epidemic.

Appetite control [18] with nutritional therapy has become of central importance, as early nutritional therapy that may assist to delay mitochondrial apoptosis connected to liver and brain disease (Figure 1) associated with diabetes and aging. A high fibre diet [19] that is low in fat and improves glucose regulation [20] activates the calorie sensitive gene Sirtuin 1 (Sirt 1) that is a $\mathrm{NAD}(+)$ dependent class III histone deacetylase protein linked to various chronic diseases [21]. Consequently, major unsolved challenges to advances in biology may now provide evidence to reverse Sirt 1 dysfunction which is connected primarily to mitochondrial apoptosis [22, 23]. Consumption of Sirt 1 activators [12] are essential to maintain Sirt 1 function in various tissues and to prevent Sirt 1 inhibitors such as food bacterial lipopolysaccharides that are associated with programmed cell death [24-28]. Novel and critical elements of scholarly peer review provide information of how the Sirt 1 is the heat shock gene and its repression is relevant to mitochondrial apoptosis in multiple organ disease syndrome, diabetes, and neurodegenerative diseases [21].

Biological active compounds/functional foods are essential to restore mitochondrial function [29] in the treatment of health and chronic disease strategies. Over-nutrition, defective caffeine metabolism, and food quality induces Sirt 1 repression [24-28] and overrides the beneficial effects of biological active food components [1, 3, 5, 29] that are essential in the treatment of global chronic disease (Figure 1).

List of Abbreviations: NAFLD, non-alcoholic fatty liver disease; Sirt 1, Sirtuin 1.

Competing Interests: Authors declare no conflicts of interest.

Acknowledgements and Funding: This work was supported by grants from Edith Cowan University, the McCusker Alzheimer's Research Foundation, and the National Health and Medical Research Council. 


\section{REFERENCES}

1. Martirosyan DM, Singh J: A new definition of functional food by FFC: what makes a new definition unique? Functional Foods in Health and Disease 2015, 5:209-223.

2. Kwak NS, Jukes, DJ: Functional foods. Part 1 . The development of a regulatory concept. Food Control 2001, 12: 99-107.

3. Hasler CM: Functional Foods: Their role in disease prevention and health promotion. Food Technology 1998, 52: 61-70.

4. Cencic A, Chingwaru W: The Role of Functional Foods, Nutraceuticals, and Food Supplements in Intestinal Health. Nutrients 2010, 2: 611-625.

5. Raikos V: Natural Antioxidants for Food Applications: Challenges and Recent Developments. EC Nutrition 2017, 8.2:33-34.

6. Gomez LM, Mesa C, Restrepo O, Duque JC, Henao JA: Our Immune System IS What We Eat. A Nutritional Immunology Approach. EC Nutrition 2015, 3.1:546-556.

7. Cox KHM, Scholey A: Polyphenols for Brain and Cognitive Health, in Recent Advances in Polyphenol Research. Edited by Yoshida K, Cheynier V, and Quideau S. Chichester, UK: John Wiley \& Sons, Ltd; 2017.

8. Vauzour D: Dietary Polyphenols as Modulators of Brain Functions: Biological Actions and Molecular Mechanisms Underpinning Their Beneficial Effects. Oxid Med Cell Longev Year 2012, 2012:1-16. http://dx.doi.org/10.1155/2012/914273.

9. Al-Dujaili E AS: Natural Polyphenols: Potential for Disease Prevention. EC Nutrition 2015, 2.2: 337-345.

10. Nonthakaew A, Matan Na, Aewsiri T Matan Ni: Caffeine in foods and its antimicrobial activity. International Food Research Journal, 2015, 22:9-14.

11. Caffeine in food and dietary supplements: Examining safety: Workshop Summary. Washington, DC: The National Academies Press. IOM (Institute of Medicine) 2014. [http://www.nap.edu/catalog.php?record_id=18607].

12. Martins IJ: Nutrition Therapy Regulates Caffeine Metabolism with Relevance to NAFLD and Induction of Type 3 Diabetes. J Diabetes Metab Disord 2017, 4: 019.

13. Martins IJ: CAFFEINE WITH LINKS TO NAFLD AND ACCELERATED BRAIN AGING. In Non-Alcoholic Fatty Liver Disease - Molecular Bases, Prevention and Treatment. Edited by Rodrigo VB. InTech - Open Science Open Minds, InTechOpen; 2017:1-24.

14. Martins IJ: Caffeine consumption with Relevance to Type 3 diabetes and accelerated brain aging. Research and Reviews: Neuroscience 2016, 1:1-5.

15. Martins IJ: Caffeine Consumption and Induction of Obesity in the Developed World. Ann Obes Disord 2017, 2:1018.

16. Eilander A, Osendarp S, Tiwari, JK: Functional Foods for the Brain, in Functional Food Product Development. Edited by Smith J and E. Charter. Oxford UK: Wiley-Blackwell; 2010.

17. Mirmiran P, Bahadoran Z, Azizi F: Functional foods-based diet as a novel dietary approach for management of type 2 diabetes and its complications: A review. World J Diabetes. 2014, 5:267-81. 
18. Martins IJ: Appetite Control with Relevance to Mitochondrial Biogenesis and Activation of Post- Prandial Lipid Metabolism in Obesity Linked Diabetes. Ann Obes Disord. 2016, 1: 1012.

19. Riccardi G, Rivellese AA: Effects of dietary fiber and carbohydrate on glucose and lipoprotein metabolism in diabetic patients. Diabeters Care 1991, 14:1115-25.

20. Martins IJ, Fernando W: High Fibre Diets and Alzheimer's Disease. FNS 2014, 5:410-424.

21. Martins IJ: Single Gene Inactivation with Implications to Diabetes and Multiple Organ Dysfunction Syndrome. J Clin Epigenet 2017, 3:1-8.

22. Martins IJ: Unhealthy Nutrigenomic Diets Accelerate NAFLD and Adiposity in Global communities. Journal of Molecular and Genetic Medicine 2015, 9: 1-11.

23. Martins IJ: Diet and Nutrition Reverse Type 3 Diabetes and Accelerated Aging linked to Global chronic diseases. J Diab Res Ther 2016, 2:1-6.

24. Martins IJ: Overnutrition Determines LPS Regulation of Mycotoxin Induced Neurotoxicity in Neurodegenerative Diseases. Int J Mol Sci 2015, 16: 29554-29573.

25. Martins IJ: Food quality induces a miscible disease with relevance to Alzheimer's disease and Neurological diseases. J Food Research 2016, 5:45-5

26. Martins IJ: Dietary Interventions Reverse Insulin and Synaptic Plasticity Defects Linking to Diabetes and Neurodegenerative Diseases. SL Nutrition and Metabolism 2017, 1:1-5.

27. Martins IJ: The Future of Genomic Medicine Involves the Maintenance of Sirtuin 1 in Global Populations. Int J Mol Biol 2017, 2: 1-4.

28. Sieniawska E: Concerns about Quality and Safety of Dietary Supplements EC. Pharmacology and Toxicology 2017,3.1:13-14.

29. Nicolson GL: Mitochondrial Dysfunction and Chronic Disease: Treatment with Natural Supplements. Integr Med (Encinitas) 2014, 13:35-43. 